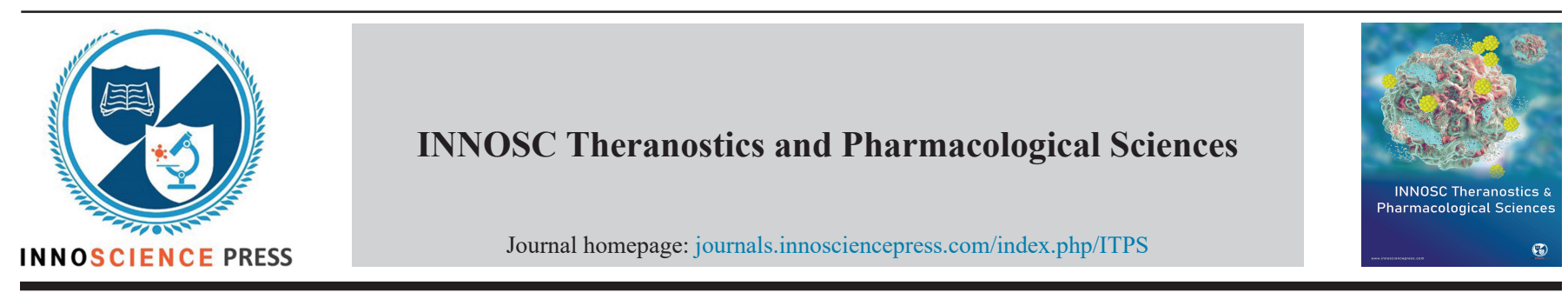

\title{
EDITORIAL
}

\section{Cardiac Surgery in the Post-COVID-19 Era}

\author{
Bessem Gara Ali, M.D.
}

Department of Cardiovascular Surgery, Bichat-Claude Bernard Hospital, Paris, France

Email: bessem_garaali@hotmail.fr

Published: September 4, 2020 DOI: 10.36922/itps.v3i1.941

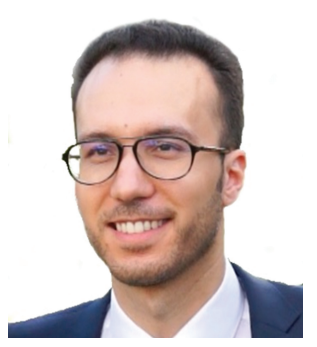

Humanity is experiencing the worst global disaster since the Second World War. On August 17, 2020, more than 21 million patients have been affected by coronavirus disease (COVID)-19 and the infectious disease has killed more than 750,000 people worldwide [1]. Since the COVID-19 outbreak, cardiac surgery was severely impacted with a drastic reduction in elective activity. Only urgent surgeries were provided to the patients with emergent needs during the peak of the pandemic. This editorial discusses the resumption of elective cardiac surgery based on the current situation.

The epidemic that initially started in Hubei Province in China at the end of December 2019 has rapidly spread to 200 countries [2]. The pandemic is getting better according to the latest figures in the United States, the most affected country, and for the rest of the world except Latin America and India [2].

Our policy as cardiac surgeons throughout the period of lockdown was to delay all elective procedures, on the one hand, and to refer patients to less invasive percutaneous coronary intervention (PCI), transcatheter aortic valve replacement (TAVR), or transcatheter mitral valve replacement (TMVR) procedures, on the other hand. Most cardiac surgeons limited surgery to cases such as acute aortic dissections and emergent coronary artery syndromes that are not amenable to PCI or time-sensitive heart valve surgery that is not amenable to TAVR or TMVR [3]. With the first signs of resolution of the pandemic and the gradual resumption of normal living in several European countries, a significant number of patients are expected to be treated, including patients with symptomatic chronic diseases who preferred not to consult during the lockdown period and asymptomatic ones who had delayed or cancelled appointments for follow-up of their chronic diseases. Elderly, overweight, hypertensive, and diabetic male patients are currently known to pay the highest price and mortality in these patients is higher than in the rest of the population [4].
Given that elective cardiac surgery activity has fallen by at least $50 \%$ according to Salenger et al., the post-COVID-19 period should result in an increase of $216-263 \%$ in the number of patients to be treated per month [5]. The period required to absorb the delay in patient management will vary between 1 and 8 months, depending on the date of resumption of full activities and the capacity of hospitals to absorb these volumes [5]. There will be coronary patients who have had an inadequately managed myocardial infarction, resulting in complications such as heart failure, myocardial aneurysm, and mitral insufficiency due to chordal or papillary muscle rupture and other mechanical complications. There will be patients suffering from severe aortic valve stenosis presenting syncope, severe dyspnea at rest or resting angina. There will also be patients with severe mitral valve insufficiency complicated by acute pulmonary edema. These complicated patients require a longer intensive care unit (ICU) stay and more resources, and have higher morbidity and mortality rates.

To be able to absorb these potentially complicated patients who were not given much attention during the pandemic, hospitals have to ensure that the necessary material and human resources are available. Unfortunately, they usually suffer from budget restrictions and are under the influence of drastic cost-cutting programs, which adds challenges to increase their capacities. With less cases being reported, more ICU beds which were previously dedicated to COVID-19 patients are now becoming available, and elective cardiac surgery activity will soon resume as usual. In addition, residents as well as fellows who have been impacted by the COVID-19 crisis should resume their residency and fellowship programs. In fact, at the peak of the pandemic, all educational and learning programs were stopped and all caregivers were assigned to the resuscitation care units dedicated to COVID-19 cases.

Given that the coronavirus may not disappear in the immediate future and a second wave is plausible, cardiac surgery 
practice must adapt to these circumstances with an emphasis on frequent hand washing, quarantine procedures, as well as social and physical distancing. All patients who are scheduled for elective surgery should be screened for COVID-19 before surgery and all patients of surgical emergency should be considered COVID-19-positive until proven otherwise. These guidelines will help break the chain of infection and protect the medical and paramedical teams. Besides, caregivers affected by the coronavirus should be quarantined for at least 2 weeks and until symptoms improve. However, this will inevitably reduce the human capacities of the cardiac surgery and postcardiac surgery resuscitation departments.

A growing number of learned societies, such as the Canadian Society of Cardiac Surgeons, have begun to issue recommendations for the post-COVID-19 era, with the goal of resumption to normal surgical activity in three phases. The first phase involves the patients with urgent surgical needs; the second phase further involves those who are less likely to require prolonged ICU and hospital length stay; and the third phase signifies the full resumption of outpatient services to normal level while continuing to prioritize those at greatest risk on the wait list [6]. During all phases, patients on the wait list for surgery should be contacted to determine their symptoms of cardiac diseases to facilitate prioritization. Programs of resumption of cardiac surgery in the post-COVID-19 era are encouraged to adopt a mechanism by which patients who are having more signs and symptoms of cardiac diseases or not doing well can contact the point of contact in the program to receive additional screening while waiting for their appointments or surgery.

\section{CONFLICTS OF INTEREST}

The author has no conflicts of interest to declare.

\section{REFERENCES}

[1] World Health Organization. COVID-19 Dashboard. Available from: https://www.covid19.who.int. [Last accessed on 2020 Aug 17].

[2] Coronavirus Tracked: The Latest Figures as the Pandemic Spreads (Free to read). Financial Times. https://www.ft.com/coronaviruslatest.

[3] Fudulu, D.P.; Angelini, G.D. Cardiac Surgery in the Time of the Coronavirus. J Card Surg., 2020, 35, 1177-9.

[4] Apicella, M.; Campopiano, M.C.; Mantuano, M.; Mazoni, L.; Coppelli, A.; Del Prato, S. COVID-19 in People with Diabetes: Understanding the Reasons for Worse Outcomes. Lancet Diabetes Endocrinol., 2020, 8, 782-92.

[5] Salenger R, Etchill EW, Ad N, Matthew T, Alejo D, Whitman G, Lawton JS, Lau CL, Gammie CF, Gammie JS, The Surge after the Surge: Cardiac Surgery post-COVID- 19. Ann. Thoracic Surg., 2020, 2020, 1-18.

[6] Hassan, A.; Arora, R.C.; Lother, S.A.; Adams, C.; Bouchard, D.; Cook, R.; Gunning, D.; Lamarche, Y.; Malas, T.; Moon, M.; Ouzounian, M.; Rao, V.; Rubens, F.; Tremblay, P.; Whitlock, R.; Moss, E.; Légaré, J.F.; Canadian Society of Cardiac Surgeons. Cardiac Surgery during the COVID-19 Pandemic: A Guidance Statement from the Canadian Society of Cardiac Surgeons. Can. J. Cardiol., 2020, 36, 952-955. 